\title{
A REVISION OF THE BERNOULLIAN AND EULERIAN FUNCTIONS *
}

BY E. T. BELL

1. Introduction. To secure simplicity and uniformity in the derivation of relations between the Bernoullian and Eulerian functions (not to be confused with the numbers of the same name) occurring most frequently in applications we take as fundamental a set of four functions instead of the usual pair, and from these by easy substitutions obtain the values of the functions defined by other writers. $\dagger$

Following Lucas $\ddagger$ we use the even suffix notation for the numbers $B, G, E, R$ of Bernoulli, Genocchi, Euler and Lucas; with the exceptions $B_{1}=-\frac{1}{2}, G_{1}=1$, the numbers of odd rank vanish, and $B_{0}=1, G_{0}=0, E_{0}=1, R_{0}=\frac{1}{2}$. Unless otherwise stated $n$ is an arbitrary integer $\geqq 0$. The "representative" or umbral calculus of Blissard § is used throughout, so that the $n$th power $a^{n}$ of the umbra $a$ represents the ordinary $a_{n}$. The letters $x, y, z, u, v$ denote ordinary algebraic quantities, or ordinaries; $a, b, c, B, E, G, R, \varphi, \psi, \lambda, \mu$ are umbræ. The umbral $\sin a x, \cos a x$ are defined by the series, assumed absolutely convergent for some $|x|>0$,

$$
\begin{aligned}
& \sin a x=\sum_{0}^{\infty}(-1)^{n} a^{2 n+1} \frac{x^{2 n+1}}{(2 n+1) !}=\sum_{0}^{\infty}(-1)^{n} a_{2 n+1} \frac{x^{2 n+1}}{(2 n+1) !}, \\
& \cos a x=\sum_{0}^{\infty}(-1)^{n} a^{2 n} \frac{x^{2 n}}{(2 n) !}=\sum_{0}^{\infty}(-1)^{n} a_{2 n} \frac{x^{2 n}}{(2 n) !} ;
\end{aligned}
$$

the umbral multinomial theorem gives the expansion of $(a x+b y+\cdots+c z)^{n}$ in either of the identical forms

* Presented to the Society, April 8, 1922.

$\dagger$ Accounts of the history and notations of these functions are contained in papers by Worpitzky, CReLle's JournaL, vol. 94 (1883), pp. 203-232, and Glaisher, QuARTERLy Journal, vol. 29 (1898), pp. 1-169; vol. 42 (1911), pp. 86-157.

$\ddagger$ Théorie des Nombres, Chap. XIV.

§ QUARTERLY JournaL, vols. 6-9 (1863-1867); cf. also Lucas, loc. cit., Chap. XIII. 


$$
\begin{aligned}
& \sum \frac{n !}{\alpha ! \beta ! \cdots \gamma !} x^{\alpha} y^{\beta} \cdots z^{\gamma} a^{\alpha} b^{\beta} \cdots c^{\gamma}, \\
& \sum \frac{n !}{\alpha ! \beta ! \cdots \gamma !} x^{\alpha} y^{\beta} \cdots z^{\gamma} a_{\alpha} b_{\beta} \cdots c_{\gamma},
\end{aligned}
$$

the $\sum$ extending to all sets of values of $\alpha, \beta, \cdots, \gamma \geqq 0$ whose sum is $n$. When precisely $r$ of the umbræ $a, b, \cdots, c$ are the same they are replaced by $r$ distinct umbræ until after the degradation of exponents. Thus $(a-a)^{2}$ is obtained from $(a-b)^{2}$ or $a^{2} b^{0}-2 a^{1} b^{1}+a^{0} b^{2}$, and its value is $2\left(a_{0} a_{2}-a_{1}^{2}\right)$. Throughout the paper $f(x)$ is an arbitrary analytic function of $x$; its derivatives with respect to $x$ are denoted by accents, $f^{\prime}(x), f^{\prime \prime}(x), \cdots$; with respect to any other variable, say $u$, of which it is a function, by $f_{u}, f_{u u}, \cdots$. As a special case of importance in applications the power series representation $k_{0}+k_{1} x+k_{2} x^{2}+\cdots$ of $f(x)$ may terminate; that is, for $m$ a finite constant, $k_{j}=0, j>m$.

2. Umbral Identities. The functions defined by

$$
\begin{aligned}
& \varphi_{n}(a, b) \equiv(a+b)^{n}+(a-b)^{n}, \\
& \psi_{n}(a, b) \equiv(a+b)^{n}-(a-b)^{n},
\end{aligned}
$$

and the identities connecting them, are fundamental for the whole subject.* Each is said to be of rank $n$. Noting that they may be written umbrally $\varphi^{n}(a, b), \psi^{n}(a, b)$, we have

$$
\begin{aligned}
& 2 \cos a x \cos b x=\cos \varphi(a, b) x, \\
& 2 \sin a x \sin b x=-\cos \psi(a, b) x ; \\
& 2 \sin a x \cos b x=\sin \varphi(a, b) x, \\
& 2 \cos a x \sin b x=\sin \psi(a, b) x .
\end{aligned}
$$

From the definitions we write down as special cases the values of $\varphi_{n}(x a, y b), \psi_{n}(x a, y b), \varphi_{n}(x, y b)$, etc. For, if $a \equiv y b$, then

$$
a_{n}=a^{n}=y^{n} b^{n}=y^{n} b_{n},
$$

since $y$ is an ordinary, $b$ an umbra; while if $a \equiv x$,

$$
a_{n}=a^{n}=x^{n} \text {. }
$$

The suffix $u$ denoting as above the $u$-derivative, we have

* This is true whatever definition of the Bernoullian and Eulerian functions be taken. Thus the existing treatments could be much simplified by the use of $\varphi, \psi$. It appears best, however, to recast the entire theory. 


$$
\lambda_{u}{ }^{n}(u, a)=n \lambda^{n-1}(u, a), \quad \lambda=\varphi, \psi ;
$$

and it is easily seen that

$$
\varphi^{n+1}(u, a)=(n+1) \int_{0}^{u} \varphi^{n}(u, a) d u+\left[1-(-1)^{n}\right] a_{n+1} .
$$

Let $\epsilon$ denote either +1 or -1 . Then

$$
\begin{aligned}
(x+\epsilon a+\epsilon b)^{n} \pm(x+\epsilon a-\epsilon b)^{n} & =(x+\epsilon \lambda(a, b))^{n} \\
& =\sum_{r=0}^{n}\left(\begin{array}{l}
n \\
r
\end{array}\right) \epsilon^{r} \lambda^{r}(a, b) x^{n-r},
\end{aligned}
$$

where $\lambda=\varphi$ or $\psi$ according as the upper or lower sign on the left is taken. Multiply this throughout by $k_{0}, k_{1}, k_{2}, \ldots$ and add all the results:

(5) $f(x+\epsilon a+\epsilon b) \pm f(x+\epsilon a-\epsilon b)=f(x+\epsilon \lambda(a, b))$,

the ambiguous sign being determined as above. In the same way,

$$
\begin{aligned}
f(x+a+b)+\epsilon_{1} f(x+a-b)+\epsilon_{2} f(x-a-b) & \\
& +\epsilon_{1} \epsilon_{2} f(x-a+b)=f(\mu(x, \lambda)),
\end{aligned}
$$

where $\lambda=\varphi(a, b)$ or $\psi(a, b)$ according as $\epsilon_{1}=+1$ or -1 , and $\mu=\varphi$ or $\psi$ according as $\epsilon_{2}=+1$ or -1 . Many more relations of a similar kind can be written down in the like manner. The process by which (5), (6) are obtained is more easily applied in many cases than the final formulas. For example, suppose we have formed the relation $(a+b)^{2 n}=c^{2 n}$. Then since $n$ is an arbitrary integer $\geqq 0$ we have identically

$$
(x+a+b)^{n}+(x-a-b)^{n}=(x+c)^{n}+(x-c)^{n},
$$

and from this, proceeding as before,

$$
f(x+a+b)+f(x-a-b)=f(x+c)+f(x-c) .
$$

All of the above formulas can be easily generalized to the case of any number $n$ of umbræ by extending (1), (2) to $n$ factors on the left. In doing this it is advantageous to take as fundamental the function generated by the product of $n_{1}$ umbral sines and $n_{2}$ umbral cosines, $n_{1}+n_{2}=n$. The cases $\left(n_{1}, n_{2}\right)=(1,1),(2,0),(0,2)$ are sufficient for the present sketch. 
From Lucas, ${ }^{*}$ we transcribe the relations

$$
\begin{aligned}
x \operatorname{ctn} x & =\cos 2 B x, \quad 2 x \tan x & =\cos 2 G x, \\
\sec x & =\cos E x, \quad x \csc x & =2 \cos R x .
\end{aligned}
$$

Although the lack of symmetry in these destroys many uniformities later we shall retain the definitions of $B_{n}, G_{n}, E_{n}$, $R_{n}$ implied in them, as Lucas' notation is well established.

3. The Fundamental Functions. The Bernoullian functions $\beta^{n}(u) \equiv \beta_{n}(u), \gamma^{n}(u) \equiv \gamma_{n}(u)$ of rank $n$ and argument $u$ are now defined by

$$
\begin{aligned}
\beta^{n}(u)=\varphi^{n}(u, 2 B) & =2 \sum_{r=0} 2^{2 r}\left(\begin{array}{c}
n \\
2 r
\end{array}\right) B_{2 r} u^{n-2 r}, \\
\gamma^{n}(u) & =\varphi^{n}(u, 2 G) ;
\end{aligned}
$$

and the Eulerian functions similarly by

$$
\begin{gathered}
\eta^{n}(u)=\varphi^{n}(u, E)=2 \sum_{r=0}\left(\begin{array}{l}
n \\
2 r
\end{array}\right) E_{2 r} u^{n-2 r}, \\
\rho^{n}(u)=\varphi^{n}(u, R) .
\end{gathered}
$$

When there can be no confusion the argument $u$ will be omitted but understood, and we shall write $\lambda_{n}(u) \equiv \lambda_{n}(\lambda=\beta, \gamma, \eta, \rho)$. The derivatives are obtained from (3),

$$
\lambda_{u}{ }^{n}=n \lambda^{n-1}, \quad(\lambda=\beta, \gamma, \eta, \rho) ;
$$

and from (9)-(12), noting the zero values of $B, G, E, R$ to write down those of $\beta, \gamma, \eta, \rho$, we have for $(\lambda, L) \equiv(\beta, B)$, $(\gamma, G)$ and $(\mu, M) \equiv(\eta, E),(\rho, R)$,

$$
\begin{aligned}
\lambda_{2 n+1} & =(2 n+1) \int \lambda_{2 n} d u, \\
\lambda_{2 n+2} & =(2 n+2) \int \lambda_{2 n+1} d u+2^{2 n+3} L_{2 n+2} ; \\
\mu_{2 n+1} & =(2 n+1) \int \mu_{2 n} d u, \\
\mu_{2 n+2} & =(2 n+2) \int \mu_{2 n+1} d u+2 M_{2 n+2} .
\end{aligned}
$$

With the initial values

$$
\text { (16) } \quad \beta_{0}=2, \quad \gamma_{0}=0, \quad \eta_{0}=2, \quad \rho_{0}=1 \text {, }
$$

* Loc. cit., p. 261. 
these enable us to calculate the successive $\lambda_{n}$ rapidly when the $B, G, E, R$ numbers are given by either tables or recurrences. From (9)-(12), (7)-(8), (1), (2) we write down the generating identities, ${ }^{*}$

(17) $\sin \beta x=2 x \operatorname{ctn} x \sin u x$, $\cos \beta x=2 x \operatorname{ctn} x \cos u x$

(18) $\sin \gamma x=4 x \tan x \sin u x$, $\cos \gamma x=4 x \tan x \cos u x$

(19) $\sin \eta x=2 \sec x \sin u x$, $\cos \eta x=2 \sec x \cos u x$; (20) $\sin \rho x=x \csc x \sin u x$, $\cos \rho x=x \csc x \cos u x$.

In conjunction with (1), (2), (7), (8) these contain the entire theory of the relations between $\beta, \gamma, \eta, \rho$. The relations are found uniformly from (17)-(20) by obvious trigonometric manipulations. There is space here only for a few examples to illustrate the processes.

4. Relations between the Functions. Multiply the identities (17) throughout by $4 x \tan x$, use the second of (7) to reduce the left-hand members of the results to the form of the corresponding member of (2), and apply (2):

$\sin \varphi(\beta, 2 G) x=8 x^{2} \sin u x, \quad \cos \varphi(\beta, 2 G) x=8 x^{2} \cos u x$.

Equate coefficients of like powers of $x$,

$$
\varphi^{n}(\beta, 2 G)=-8 \frac{d^{2} u^{n}}{d u^{2}}
$$

generalize this as in obtaining (5), noticing that $f^{\prime \prime}(x+u)$ $=f_{u u}(x+u)$ :

(21) $f(x+\beta+2 G)+f(x+\beta-2 G)=-8 f^{\prime \prime}(x+u)$.

Similarly from (18),

(22) $f(x+\gamma+2 B)+f(x+\gamma-2 B)=-8 f^{\prime \prime}(x+u)$.

If in this $f(x) \equiv x^{n}$, and then $x=0$, the form from which it was inferred is recovered. In the same way from (19), (20) after multiplication throughout by $2 \cos x, 2 \sin x$ respectively,

$$
\begin{aligned}
& f(x+\eta+1)+f(x+\eta-1)=4 f(x+u), \\
& f(x+\rho+1)-f(x+\rho-1)=2 f^{\prime}(x+u) ;
\end{aligned}
$$

and from (17), (18) for the multipliers $\sin x, \cos x$ we find first

$$
\psi^{n}(\beta, 1)=2 \varphi_{u}^{n}(u, 1), \quad \varphi^{n}(\gamma, 1)=-4 \psi_{u}^{n}(u, 1),
$$

* Note the unnecessary lack of symmetry caused by the unfortunate definitions (7), (8). 
whence by generalization to functions $f$,

(25) $f(x+\beta+1)-f(x+\beta-1)=2 f^{\prime}(x+u+1)+2 f^{\prime}(x+u-1)$,

(26) $f(x+\gamma+1)+f(x+\gamma-1)=4 f^{\prime}(x+u-1)-4 f^{\prime}(x+u+1)$.

Again, dividing each of the identities in each of (17)-(20) by the other we get, for $\lambda=\beta, \gamma, \eta, \rho$,

$\cos \lambda x \tan u x=\sin \lambda x, \quad \sin \lambda x \cot u x=\cos \lambda x$,

and hence as before,

$$
\varphi^{2 n}(\lambda, 2 G u)=-4 u \lambda_{u}^{2 n}, \quad \varphi^{2 n+1}(\lambda, 2 B u)=2 u \lambda_{u}{ }^{2 n+1} .
$$

These express the functions of given rank linearly in terms of functions of the same kind and lower ranks. To generalize we proceed as in $\S 2$, getting

$$
\begin{aligned}
& f(x+\lambda+2 G u)+f(x-\lambda-2 G u)-\lambda(x-\lambda+2 G u) \\
&+\left.f(x+\lambda-2 G u)+f(x-\lambda)+f_{u}(x-\lambda)\right], \\
&=-4 u\left[f_{u}(x+\lambda)\right. \\
& f(x+\lambda+2 B u)-f(x-\lambda-2 B u) \\
&+f(x+\lambda-2 B u)-f(x-\lambda+2 B u) \\
&=2 u\left[f_{u}(x+\lambda)-f_{u}(x-\lambda)\right],
\end{aligned}
$$

which hold for $\lambda=\beta, \gamma, \eta, \rho$. The identities from which these are inferred may be recovered as indicated above.

A relation between functions $f$ whose arguments contain at most $r$ distinct umbræ chosen from among $\beta, \gamma, \eta, \rho$ is called $r$-fo'd. It would be of interest to write out a set of such relations for $r=1,2,3,4$ containing $4,6,4,1$ relations of the respective types (the case $r=1$ is treated above); but to save space we indicate the method for $r>1$ in one case only. When $r=2$ the simplest relations are found by multiplying together two of the generating identities and proceeding as above. Thus (19), (20) give

$$
\begin{aligned}
& \cos \psi(\eta, \rho) x=-4 \cos 2 R x+2 \cos 2 \rho x, \\
& \cos \varphi(\eta, \rho) x=4 \cos 2 R x+2 \cos 2 \rho x ;
\end{aligned}
$$

and hence

$$
(\eta \pm \rho)^{2 n}=2^{2 n+1} \rho^{2 n}, \quad 2^{2 n+2} R^{2 n},
$$

the upper sign giving the first on the right, the lower the second. Generalizing we get 


$$
\begin{aligned}
& f(x+\eta+\rho)+f(x-\eta-\rho)=2 f(x+2 \rho)+2 f(x-2 \rho), \\
& f(x+\eta-\rho)+f(x-\eta+\rho)=4 f(x+2 R)+4 f(x-2 R) .
\end{aligned}
$$

Other simple types arise from division; thus

$$
f(x+\eta+2 B)+f(x+\eta-2 B)=4 f(x+\rho) .
$$

5. Functions of Argument $2 u, 2 u-1$. In many applications the values of the functions in $\S 3$ are required only for the arguments $2 u, 2 u-1$. It is then desirable to consider the functions of $2 u, 2 u-1$ as new functions of $u$. Accordingly we write the definitions

$$
\lambda_{j}^{n}(u) \equiv \lambda_{j, n}(u)=\lambda^{n}(2 u-j), \quad(j=0,1),
$$

for $\lambda=\beta, \gamma, \eta, \rho$. There can be no confusion between the constant suffix $j$ and the $u$ in $\lambda_{j u}{ }^{n}$ denoting the $u$-derivative of $\lambda_{j}{ }^{n}$ (the argument $u$ being omitted as before). We have $\lambda_{j}^{n}(u)=(\lambda(2 u)-j)^{n}$, as is evident on expanding by Taylor's theorem and noting (3). The generating identities are written down from (17)-(20) by changing $u$ into $2 u-j$ and replacing $\lambda$ by $\lambda_{j}$. Hence all the relations between the $\lambda_{j}{ }^{n}(j=0,1)$ functions of either kind can be written down immediately from those for the $\lambda^{n}$ functions by replacing in the latter relations $\lambda$ by $\lambda_{j}$ and $u$ by $2 u$ or $2 u-1$ according as $j=0$ or 1 . It is therefore unnecessary to repeat any of the derivations.

We note one relation of particular interest, the analogue for the $\lambda_{1}{ }^{n}(u)(\lambda=\beta, \gamma, \eta, \rho)$ of relations found by Raabe, Schlömilch and Glaisher for their functions, the obviousness of which by the symbolic method is striking:

$$
\lambda_{1}{ }^{n}(u)=(-1)^{n} \lambda_{1}{ }^{n}(1-u), \quad(\lambda=\beta, \gamma, \eta, \rho) .
$$

Another type may be noticed. Expand $\sin (2 u-1) x$, $\cos (2 u-1) x$ in the generating relations by the addition theorems for the sine and cosine, use (17), (18) to reduce the results, and finally equate coefficients of like powers of $x$. Thus

$$
\begin{aligned}
\sin \beta_{1} x & =2 x \operatorname{ctn} x[\sin 2 u x \cos x-\cos 2 u x \sin x] \\
& =2 x \csc x \sin 2 u x-2 x \cos (2 u-1) x, \\
& =2 \sin \rho(2 u) x-2 x \cos (2 u-1) x,
\end{aligned}
$$

and similarly for $\cos \beta_{1} x$ and the rest. We find hence 


$$
\begin{aligned}
\beta_{1}^{n}(u) & =2 \rho^{n}(2 u)-2 n(2 u-1)^{n-1}, \\
\gamma_{1}^{n}(u) & =-2 n \eta^{n-1}(2 u)+4 n(2 u-1)^{n-1}, \\
2 n \eta_{1}{ }^{n-1}(u) & =\gamma^{n}(2 u)+4 n(2 u)^{n-1}, \\
2 \rho_{1}{ }^{n}(u) & =\beta^{n}(2 u)-2 n(2 u)^{n-1} ;
\end{aligned}
$$

and generalizing,

$$
\begin{aligned}
f\left(x+\beta_{1}\right) & =2 f\left(x+\rho_{0}\right)-f_{u}(x+2 u-1), \\
f\left(x+\gamma_{1}\right) & =-f_{u}\left(x+\eta_{0}\right)+2 f_{u}(x+2 u-1), \\
f_{u}\left(x+\eta_{1}\right) & =f\left(x+\gamma_{0}\right)+2 f_{u}(x+2 u), \\
2 f\left(x+\rho_{1}\right) & =f\left(x+\beta_{0}\right)-f_{u}(x+2 u) .
\end{aligned}
$$

Thus if a relation between any members of either set $\lambda_{j}$ $(\lambda=\beta, \gamma, \eta, \rho, j=0,1)$ be given, a relation between the corresponding members of the other set can be written down from it, $\beta, \rho$ and $\gamma, \eta$ being the pairs of correspondents.

6. Values of the $\lambda_{j}{ }^{n}(j=0,1)$ for Integral Arguments $>0$. The connection between our functions and those of other writers is obtained (among other ways) by noting the sums of like powers of integers to which the $\lambda_{j}{ }^{n}$ degenerate when the argument $u$ is an integer $>0$. There are 16 cases in sets of four each, obtained by multiplying $\sin 2 u x, \cos 2 u x$, sin $(2 u-1) x, \cos (2 u-1) x$ by $x \operatorname{ctn} x, 2 x \tan x, \sec x, x \csc x$, reducing the products to linear functions of circular functions (of which not more than one in any case is other than a sine or cosine), and taking the coefficient of $x^{2 n} /(2 n)$ ! or $x^{2 n+1} /(2 n+1)$ ! according as the expanded product is an even or an odd function of $x$. These coefficients are simply expressible in terms of $\lambda_{j}(j=0,1)$ functions; they also are readily expressed in terms of the Bernoullian and Eulerian functions already in the literature. A full discussion of them having been given elsewhere it suffices here to state the reference.*

UNIVERSITY OF WASHINGTON

* Messenger of Mathematics, vol. 50 (1921), pp. 177-186. The functions $\varphi, \psi$ of the paper differ slightly from those of the present, also $\beta, \gamma$ there have no connection with $\beta, \gamma$ here. 